\title{
Progress towards Accurate Quantitative Standardless X-ray Analysis at Low kV
}

\author{
P. J. Statham \\ Oxford Instruments NanoAnalysis, High Wycombe, Bucks HP12 3SE, U.K.
}

At $20 \mathrm{kV}$, quantitative analysis with relative errors below $5 \%$ can be delivered by EDS standardless analysis provided SDD detector efficiency is accurately determined and standards have been pre-measured on a well-characterised detector [1]. The increasing need to analyse features well below $1 \mu \mathrm{m}$ in size has encouraged use of lower beam voltages. At $5 \mathrm{kV}$, there is at least one accessible line emission for most elements and an expectation that electron probe analysis can deliver similar accuracy. However, esoteric problems with low energy emissions, some identified years ago by WDS experts, need to be addressed such as when using transition element L lines [2]. Even before X-ray corrections are considered, spectral integrity is of paramount importance. At low $\mathrm{kV}$, large area SDDs can collect high count rates that are concentrated in fewer peaks and exacerbate pile-up probability. Even "triple sum peaks", where the original pulse is followed by two pulses within the resolving time, can be significant. A new algorithm has been developed that removes both double and triple sum peaks and corrects for counting losses as fast as the previous single pile-up correction [3]; an example is shown in figure 1. The standard procedure for stripping escape peaks [4] causes a bipolar residual because the escape peak at (E-1.74) $\mathrm{keV}$ is narrower than its parent at $\mathrm{E} \mathrm{keV}$. By resolution-enhancing the predicted escape spectrum prior to stripping, artefacts that introduce area errors are avoided (fig.2).

Accurate peak shapes are essential to determine relative peak intensities in an unresolved overlap [5]. The peak response to a single line emission has been modelled after checking that electronic defects such as ballistic deficit and amplifier non-linearity have been eliminated. Incomplete charge collection causes complex energy-dependent changes in position and resolution; a new parameterisation corrects these typically to within $1 \mathrm{eV}$. Published line emission tables do not give accurate relative intensities because these depend on specimen and excitation. Absorption effects can produce a dramatic difference in the EDS peak profile at 5 and $20 \mathrm{kV}$ as shown in fig.3. Even where absorption is not significant, differential excitation of $\mathrm{L}$ sub-shells and Coster-Kronig enhancement can give rise to unexpected profile changes as shown for Ag in fig.4. With the improved line response function and corrections for differential absorption and excitation, we used the data obtained at different $\mathrm{kV}$ to optimise relative line intensities for all lines in $\mathrm{K}, \mathrm{L}$ and $\mathrm{M}$ series and deduce empirical mass absorption coefficients.

With accurate efficiency calibration, improved characterisation and new line data, accurate peak profiles can be generated for any $\mathrm{kV}$ without recourse to standards. Full quantitative analysis can also be achieved with only a single pure element reference measurement. At low $\mathrm{kV}$, superficial carbon and oxygen layers can introduce errors. Fig.5 shows effects seen on an old standards block for several element lines measured at $5 \mathrm{kV}$. While intensities are typically reduced by $5-20 \%$, surface layers roughly halve intensity for Sc K, which has low over-voltage. Quantitative accuracy is therefore improved by using a correction for superficial layers (AZtec LayerProbe using Stratagem/XPP).

\section{References}

[1] P.J.Statham (2010) Microscopy and Microanalysis, 16 (Suppl. 2), 1304-1305

[2] P.J.Statham (2013) Microscopy and Microanalysis, 19 (Suppl. 2), 1246-1247

[3] P.J.Statham (2006) Microchim Acta 155, 289-294 
[4] S.J.B. Reed and N.G. Ware, (1972) J. Phys. E: Sci. Instr. 5, 582-584

[5] P. J. Statham (2002) J. Res. Natl. Inst. Stand. Technol. 107, 531-546

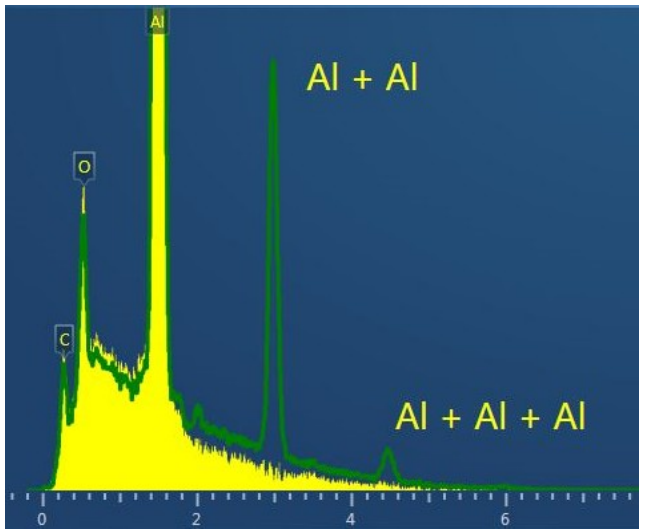

F IG. $1.5 \mathrm{kV}$ Al spectrum at $300 \mathrm{kcps}$ input rate (green) and pile-up corrected spectrum (yellow).

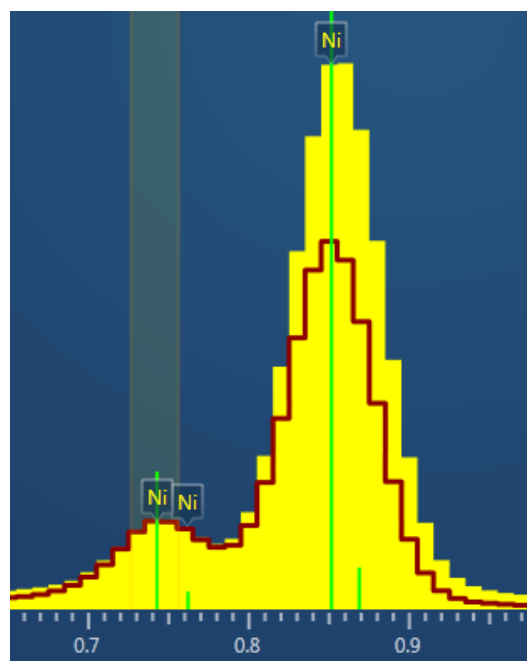

FIG. 3. Measured spectra from pure Ni at $5 \mathrm{kV}$ (yellow) and $20 \mathrm{kV}$ (red)
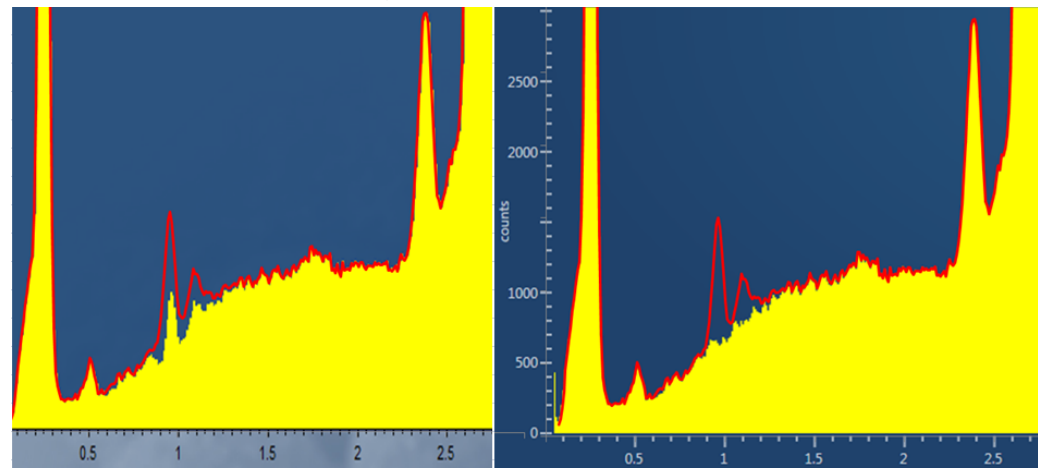

FIG. 2. Correction for Si K escape peak artefact from $\mathrm{Rh} \mathrm{L}$ parent. Red line is uncorrected spectrum. Corrected yellow bars show standard (left) and resolution-corrected (right) escape peak strip.

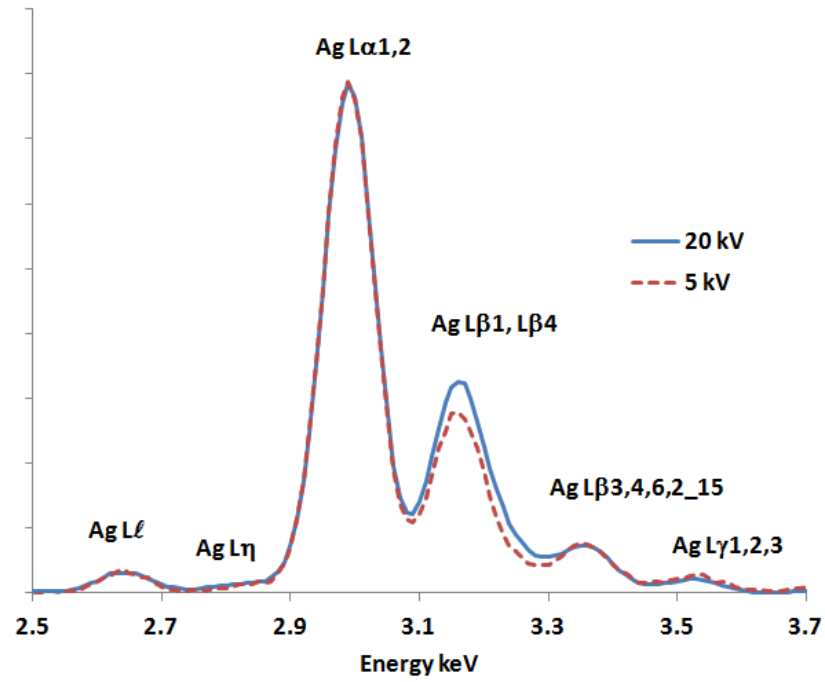

FIG. 4. Measured spectra of pure $\mathrm{Ag}$ at $5 \mathrm{kV}$ and $20 \mathrm{kV}$, background-corrected, scaled to match at main peak.

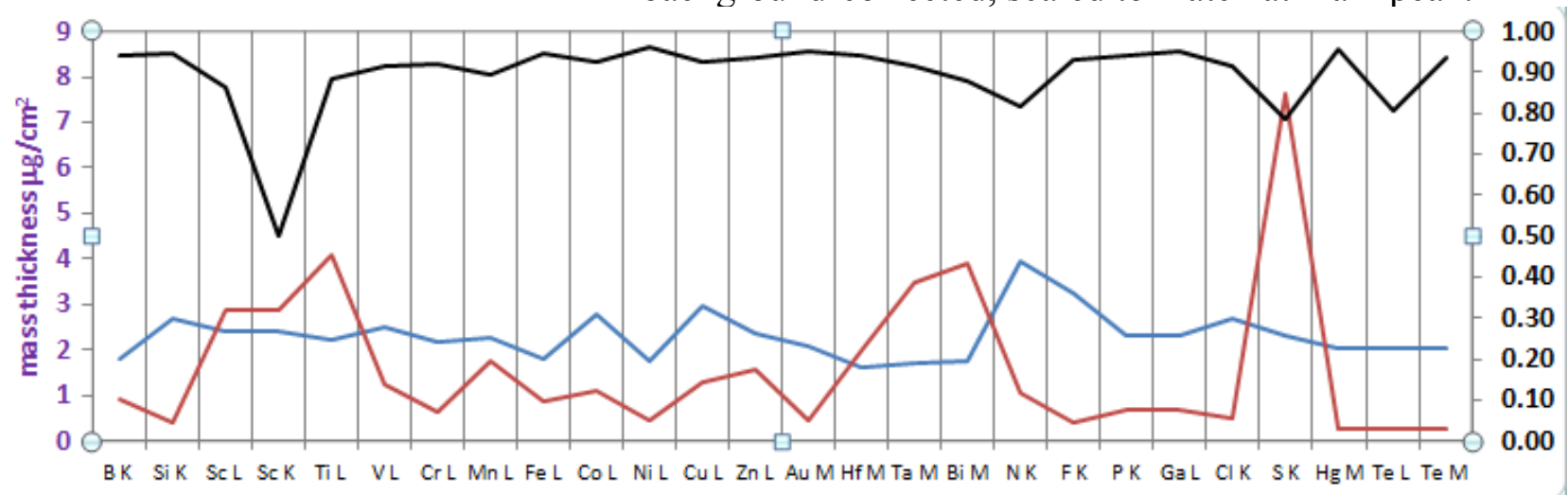

FIG. 5. Measured C (blue) and O (red) mass thickness $\mu \mathrm{g} / \mathrm{cm}^{2}$ for standards on an old standards block. Black line (scale on right) shows effect of these layers as the factor reducing intensity at $5 \mathrm{kV}$ 